\title{
Analytical solution for phase space evolution of electrons operating in a self-amplified spontaneous emission free electron laser
}

\author{
Nobuyuki Nishimori* \\ Advanced Photon Source Center, Japan Atomic Energy Agency (JAEA), Tokai, Naka, Ibaraki 319-1195, Japan
}

(Received 1 January 2005; published 11 October 2005)

\begin{abstract}
I present an analytical solution for the phase space evolution of electrons in a self-amplified spontaneous emission (SASE) free-electron laser (FEL) operating in the linear regime before saturation in the resonant case by solving the one dimensional FEL equation together with the solution of the cubic equation, which represents the evolution of the SASE FEL field. The electrons are shown to be bunched around $\pi / 6$ ahead of a resonant electron every resonant FEL wavelength in the high gain regime. The phase relation is similar to that in a low gain FEL where an electron beam above resonance is injected, explaining the positive FEL gain. The analytical solutions agree well with numerical simulations and are applied to obtain the coherent optical transition radiation (OTR) intensity produced from electron microbunching at FEL wavelength. The coherent OTR intensity is shown to be proportional to FEL intensity.
\end{abstract}

DOI: 10.1103/PhysRevSTAB.8.100701

PACS numbers: $41.60 . \mathrm{Cr}$

\section{INTRODUCTION}

A self-amplified spontaneous emission (SASE) freeelectron laser (FEL) has been developed worldwide as an intense coherent $\mathrm{x}$-ray radiation source [1-3]. The development has been supported by extensive theoretical studies [4-7], which can account for various types of experimental results such as the exponential increase of SASE power with the undulator length [1]. This exponential growth of SASE FEL power can be represented by the solution of the cubic equation, which is derived from one dimensional (1D) FEL equations representing both the electron dynamics in the laser field and the dynamics of the FEL field $[4,6,8]$. Those theoretical studies have however focused on the property of the radiation field, and the phase space evolution of electrons of a SASE FEL has been studied only in numerical simulations so far [7].

In this paper, I present an analytical solution for the phase space evolution of electrons in a SASE FEL operating in the linear regime before saturation by solving the 1D equations of electron motion together with the solution of the cubic equation in the resonant case [4], which represents the evolution of SASE FEL field. The solutions for the energy and phase changes of electrons are, respectively, represented by the sum of three independent terms similarly to the solution of the cubic equation; an exponentially growing term, an exponentially decaying term and an oscillating term (see Sec. III). The electrons are shown to be bunched around $\pi / 6$ ahead of a resonant electron every resonant FEL wavelength in the high gain regime. This phase relation is similar to that in a low gain FEL where an electron beam above resonance is injected, explaining the positive FEL gain. Substitution of the solution into the 1D Maxwell equation yields the same field gain as that derived

*Electronic address: nishimori.nobuyuki@jaea.go.jp from the solution of the cubic equation. The solutions for the phase space evolution are thus complementary to the solution for the optical evolution and agree well with a time-dependent numerical simulation starting from a shot noise of electrons and solving the 1D FEL equation (see Sec. V). The saturation behavior is studied by a numerical calculation which solves the 1D FEL equations with the initial values given by the present analytical solutions, as shown in Sec. IV. The solutions are also applied to study the coherent optical transition radiation (OTR) produced from electron microbunching at FEL wavelength in Sec. VI. The coherent OTR intensity is shown to be proportional to FEL intensity, which can account for the undulator length dependence of the coherent OTR from electron beam microbunching observed in a SASE FEL at Argonne Photon Source (APS) [9].

\section{1D FEL EQUATION}

When a relativistic electron beam travels through an undulator, it emits spontaneous radiation along the undulator axis. The bandwidth of the spontaneous radiation decreases as the beam passes through the undulator because of the spectrum narrowing [8]. The successive electric interaction between the radiation field and the undulating electrons leads to further amplification of the radiation field. The electron dynamics in the radiation field and the dynamics of the laser field are given by 1D FEL equations $[8,10]$.

The dimensionless 1D FEL equations by Colson is used in the present study under the slowly varying envelope approximation (SVEA) [11], while the variables used here are similar to Bonifacio's variables [6]. The simplest situation is considered in the present study. The electron beam energy is given by $\gamma_{0} m c^{2}$ with small energy spread. The initial electron pulse has a rectangular shape with 
density of $n_{e}$ and a uniform distribution in phase. The electron pulse length is assumed to be longer than the slippage distance $N_{w} \lambda_{r}$. Here $N_{w}$ is the number of undulator periods, $\lambda_{r}=\lambda_{w}\left(1+a_{w}^{2}\right) /\left(2 \gamma_{0}^{2}\right)$ is the resonant wavelength, $\lambda_{w}=2 \pi / k_{w}$ is the period of the undulator, and $a_{w}$ is the undulator parameter. The fundamental FEL parameter in MKSA units is given by

$$
\rho=\frac{1}{\gamma_{0}}\left[e a_{w} F \sqrt{n_{e} /\left(\epsilon_{0} m\right)} /\left(4 c k_{w}\right)\right]^{2 / 3},
$$

where $F$ is unity for a helical undulator or Bessel function $[J J]$ for a planar undulator [6]. The dimensionless time is defined by $\tau=(4 \pi \rho) c t / \lambda_{w}$, so that $\delta \tau=1$ corresponds to the transit time of light through one gain length of $\lambda_{w} /(4 \pi \rho)$. The longitudinal position of the $i$ th electron is defined by $\zeta_{i}(\tau)=(4 \pi \rho)\left[z_{i}(t)-c t\right] / \lambda_{r}$, so that $\delta \zeta=1$ corresponds to the cooperation length defined by $\lambda_{r} /(4 \pi \rho)$. The dimensionless field envelope is defined by

$$
a(\zeta, \tau)=\frac{2 \pi e a_{w} \lambda_{w} F}{(4 \pi \rho)^{2} \gamma_{0}^{2} m c^{2}} E(\zeta, \tau) \exp [i \phi(\zeta, \tau)]
$$

with phase $\phi(\zeta, \tau)$, which is equivalent to the Bonifacio's envelope [6]. Here $E(\zeta, \tau)$ is the rms optical field strength. The dimensionless energy and phase of the $i$ th electron are, respectively, defined by $\mu_{i}(\tau)=\left[\gamma_{i}(t)-\gamma_{0}\right] /\left(\rho \gamma_{0}\right)$ and $\psi_{i}(\tau)=\left(k_{w}+k_{r}\right) z_{i}(t)-\omega_{r} t$, where $k_{r}=2 \pi / \lambda_{r}$ is the wave number of the resonant wavelength $\lambda_{r}$. The dimensionless energy $\mu_{i}(\tau)$ also means dimensionless energy change at $\tau$ from $\tau=0$, since the energy spread of the initial electron beam is assumed to be small, i.e., $\mu_{i}(0)=0$.

In the present definition, evolutions of the field envelope $a(\zeta, \tau)$, the energy $\mu_{i}(\tau)$ and phase $\psi_{i}(\tau)$ of the $i$ th electron during FEL interaction are, respectively, given by [8]

$$
\begin{gathered}
\frac{d \mu_{i}(\tau)}{d \tau}=a\left[\zeta_{i}(\tau), \tau\right] \exp \left[i \psi_{i}(\tau)\right]+\text { c.c., } \\
\frac{d \psi_{i}(\tau)}{d \tau}=\mu_{i}(\tau) \\
\frac{\partial a(\zeta, \tau)}{\partial \tau}=-\left\langle\exp \left[-i \psi_{i}(\tau)\right]\right\rangle_{\zeta_{i}(\tau)=\zeta}
\end{gathered}
$$

The angular bracket indicates the average of all the electrons in the volume $V$ around $\zeta$.

\section{PHASE SPACE EVOLUTION}

The lasing process in FELs starts with formation of a uniform field in time and space. This process is known as the spectrum narrowing in the frequency domain [5] or as the longitudinal phase mixing in the time domain [12]. The phase of the field $\phi(0)$ is almost uniform over length $N \lambda_{r}$ along the propagation direction when the incident electron beam passes through $N$ undulator periods $[8,12]$. In the present study, the initial uniform field is assumed to be given by $|a(0)| e^{i \phi(0)}$ for simplicity, where $|a(0)|$ is almost equal to the amplitude of spontaneous radiation emitted in order of 1 gain length [5]. In other words, the uniform field is assumed to be established at $\tau=0$ without spectrum narrowing in the present study. The initial field evolves through electric interaction with undulating electrons as it passes through the undulator. The incident electron beam is assumed to be uniformly distributed in phase $\psi_{i}(0)$ with resonant energy $\mu_{i}(0)=0$, as described in the previous section. The evolution of the uniform field as a function of time is derived from Eqs. (3)-(5), as described by Colson et al. in Ref. [13].

The electron phase can be expressed as $\psi_{i}(\tau)=\psi_{i}(0)+$ $\Delta \psi_{i}(\tau)$ where $\Delta \psi_{i}(\tau)$ is the first order perturbation in $a(\tau)$. The field at time $\tau$ for the steady-state region where $\zeta<$ $-\tau$ is given by

$$
a(\tau)=a(0)+i \int_{0}^{\tau}\left\langle\exp \left[-i \psi_{i}(0)\right] \Delta \psi_{i}\left(\tau^{\prime}\right)\right\rangle_{\zeta_{i}(\tau)=\zeta} d \tau^{\prime}
$$

The $i$ th electron interacts with the field in the steady-state region due to the slippage, and the energy modulation at $\tau^{\prime}$ during $\delta \tau^{\prime}$ is given from Eq. (3) by $\delta \mu_{i}\left(\tau^{\prime}\right)=$ $\left[a\left(\tau^{\prime}\right) e^{i \psi_{i}(0)}+\right.$ c.c. $] \delta \tau^{\prime}$. The energy change of the $i$ th electron at time $\tau, \mu_{i}(\tau)$, is given by the sum of those modulations during $\tau$ :

$$
\mu_{i}(\tau)=\int_{0}^{\tau}\left\{a\left(\tau^{\prime}\right) \exp \left[i \psi_{i}(0)\right]+\text { c.c. }\right\} d \tau^{\prime} .
$$

The electron phase perturbation is given from Eq. (4) by

$$
\begin{gathered}
\Delta \psi_{i}(\tau)=\int_{0}^{\tau} \mu_{i}\left(\tau^{\prime}\right) d \tau^{\prime} \\
=\int_{0}^{\tau} d \tau^{\prime} \int_{0}^{\tau^{\prime}}\left\{a\left(\tau^{\prime \prime}\right) \exp \left[i \psi_{i}(0)\right]+\text { c.c. }\right\} d \tau^{\prime \prime}
\end{gathered}
$$

Substitution of Eq. (9) into Eq. (6) leads to

$$
a(\tau)=a(0)+i \int_{0}^{\tau} d \tau^{\prime} \int_{0}^{\tau^{\prime}} d \tau^{\prime \prime} \int_{0}^{\tau^{\prime \prime}} a\left(\tau^{\prime \prime \prime}\right) d \tau^{\prime \prime \prime} .
$$

The integral Eq. (10) can be written in a differential form by taking successive derivatives, $\dddot{a}(\tau)=i a(\tau)$. The solution is expressed by the form $a(\tau)=\sum_{n=1}^{3} a_{n} \exp \left(\alpha_{n} \tau\right)$ where the $\alpha_{n}$ are three complex roots of the cubic equation $\alpha^{3}=i[4,6,13]$. When the initial conditions $\dot{a}(0)=$ $\ddot{a}(0)=0$, the field at time $\tau$ for the steady-state region where $\zeta<-\tau$ is given by

$$
\begin{aligned}
a(\tau)= & \frac{|a(0)| e^{i \phi(0)}}{3}\left[\exp \left(\tau e^{i \pi / 6}\right)+\exp \left(-\tau e^{-i \pi / 6}\right)\right. \\
& \left.+\exp \left(\tau e^{-i \pi / 2}\right)\right],
\end{aligned}
$$

where the first term is an exponentially growing term, the second term is an exponentially decaying term, and the third is an oscillating term. Equation (11) is valid in the 
linear regime before saturation when the incident electron beam is resonant.

The integration of Eq. (7) after substitution of Eq. (11) yields

$$
\begin{aligned}
\mu_{i}(\tau)= & \frac{2|a(0)|}{3}\left\{e^{\sqrt{3} \tau / 2} \cos \left[\psi_{i}(0)+\phi(0)+\tau / 2-\pi / 6\right]\right. \\
& -e^{-\sqrt{3} \tau / 2} \cos \left[\psi_{i}(0)+\phi(0)+\tau / 2+\pi / 6\right] \\
& \left.+\cos \left[\psi_{i}(0)+\phi(0)-\tau+\pi / 2\right]\right\} .
\end{aligned}
$$

The first term in the right hand side of Eq. (12) is the exponentially growing term, the second is the exponentially decaying term, and the third is the oscillating term. The integration of Eq. (8) after substitution of Eq. (12) yields

$$
\begin{aligned}
\Delta \psi_{i}(\tau)= & \frac{2|a(0)|}{3}\left\{e^{\sqrt{3} \tau / 2} \cos \left[\psi_{i}(0)+\phi(0)+\tau / 2-\pi / 3\right]\right. \\
& +e^{-\sqrt{3} \tau / 2} \cos \left[\psi_{i}(0)+\phi(0)+\tau / 2+\pi / 3\right] \\
& \left.+\cos \left[\psi_{i}(0)+\phi(0)-\tau+\pi\right]\right\} .
\end{aligned}
$$

Equations (12) and (13) are the analytical expressions for the phase space evolution in a SASE FEL operating in the linear regime; only numerical solutions for those have been obtained previously [7]. The gain of the steady-state field at time $\tau$ is obtained by substitution of Eq. (13) into Eq. (5) as follows:

$$
\begin{aligned}
\frac{d a(\tau)}{d \tau}= & \frac{|a(0)| e^{i \phi(0)}}{3}\left[\exp \left(\tau e^{i \pi / 6}\right) e^{i \pi / 6}\right. \\
& \left.-\exp \left(-\tau e^{-i \pi / 6}\right) e^{-i \pi / 6}+\exp \left(\tau e^{-i \pi / 2}\right) e^{-i \pi / 2}\right]
\end{aligned}
$$

when $\left|\Delta \psi_{i}(\tau)\right| \ll 1$. Equation (14) is the same as differentiation of Eq. (11) with respect to $\tau$.

The longitudinal phase space distribution of electrons within a resonant wavelength is usually depicted in a phase space composed of phase $\psi_{i}(\tau)$ and energy $\mu_{i}(\tau)[7,8]$. This phase space is suitable for one to see how electron microbunching develops via the longitudinal ponderomotive force, but not to see how much the individual electron moves from its initial phase, especially in the early stage of the FEL evolution because of $\Delta \psi_{i}(\tau) \ll 1$. A phase space composed of phase change $\Delta \psi_{i}(\tau)$ and energy change $\mu_{i}(\tau)$ allows one to see the time evolution of the electron microbunching.

In the present paper, the electrons at $\tau=0$ are numbered depending on their longitudinal positions from the front to the rear. The relative position between two adjacent electrons is thus represented by $z_{i}(0)>z_{i+1}(0)$, and the relative phase is given by $\psi_{i}(0)>\psi_{i+1}(0)$ by definition. One can calculate the values of Eqs. (12) and (13) for each $i$ and plot the point in the phase space, as shown in Fig. 1. The electrons within resonant wavelength $\lambda_{r}$ are distributed along an ellipse at time instant $\tau$ and are lined along the

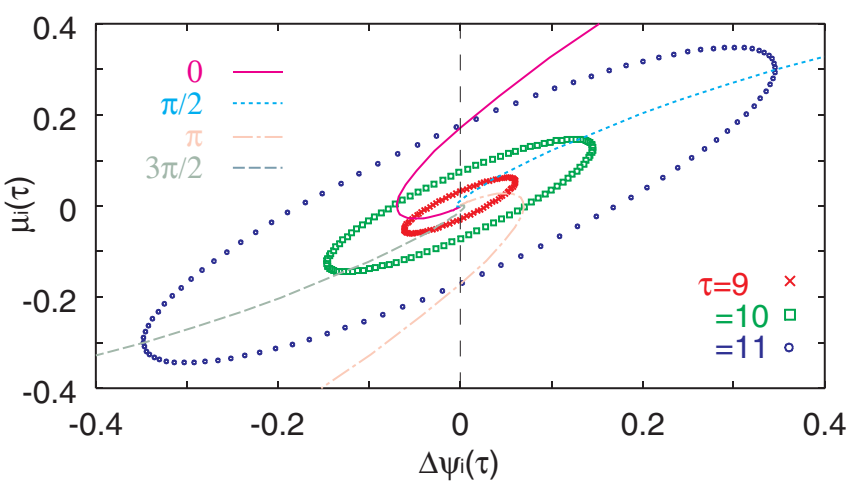

FIG. 1. (Color) Electron distributions in a phase plane of $\Delta \psi_{i}(\tau)$ and $\mu_{i}(\tau)$ derived from Eqs. (12) and (13) when $\tau=9$ (crosses), 10 (open squares), 11 (open circles). The time evolution of four different electrons, initial phases of which are $\psi_{i}(0)$ (solid line), $\psi_{i}(0)+\pi / 2$ (dotted line), $\psi_{i}(0)+\pi$ (dash-dotted line), and $\psi_{i}(0)+3 \pi / 2$ (dashed line), are also shown. The initial amplitude of the radiation $|a(0)|=3.79 \times 10^{-5}$ is used.

ellipse counterclockwise as the identification number $i$ increases. The crosses are the distribution when $\tau=9$, the open squares are when $\tau=10$ and the open circles are when $\tau=11$. The figure also shows the time evolution of four electron particles, initial phases of which are $\psi_{i}(0)$ (solid line), $\psi_{i}(0)+\pi / 2$ (dotted line), $\psi_{i}(0)+\pi$ (dashdotted line), and $\psi_{i}(0)+3 \pi / 2$ (dashed line). One can find that the ellipse expands exponentially in size and rotates clockwise with time. In the calculation $|a(0)|=$ $3.79 \times 10^{-5}$ is used. This value is the same as that used in the simulation which will be described in Sec. V.

The center of the microbunch is the place where electrons within resonant wavelength $\lambda_{r}$ are concentrated every resonant FEL wavelength. The electron at the microbunch center satisfies the condition of $\Delta \psi_{c}(\tau)=0$, since $\Delta \psi_{i}(\tau)=-\Delta \psi_{j}(\tau)$ when $\psi_{i}(0)=\psi_{c}(0)+\delta \theta$ and $\psi_{j}(0)=\psi_{c}(0)-\delta \theta$ from Eq. (13). The $(c-1)$ th electron which is located just in front of the microbunch center, that is the $c$ th electron, satisfies the condition of $\Delta \psi_{c-1}(\tau)<0$ and the $(c+1)$ th electron satisfies the condition of $\Delta \psi_{c+1}(\tau)>0$. The intersection of the ellipse and the line $\Delta \psi_{i}(\tau)=0$ where $\mu_{i}(\tau)<0$ is the location of the microbunch center. Figure 2 depicts electron distributions corresponding to those shown in Fig. 1 in a usual longitudinal phase space of $\psi_{i}(\tau)$ and $\mu_{i}(\tau)$. The bunch center satisfying $\Delta \psi_{i}(\tau)=0$ is indicated by an arrow and located $\pi / 6$ ahead of a resonant electron $\mu_{i}(\tau)=0$, as supported by Eqs. (12) and (13) when $\tau \geq 4$. This phase difference between $\Delta \psi_{i}(\tau)=0$ and $\mu_{i}(\tau)=0$ can be attributed to the facts that modulation in phase requires an additional time after modulation in energy similarly to a buncher section in an accelerator system and that the electron beam is continuously modulated in energy inside an undulator. The phase relation between $\Delta \psi_{i}(\tau)=0$ and $\mu_{i}(\tau)=0$ is similar to the low gain FEL with an incident electron beam above resonance $[8,10]$. This ensures that 


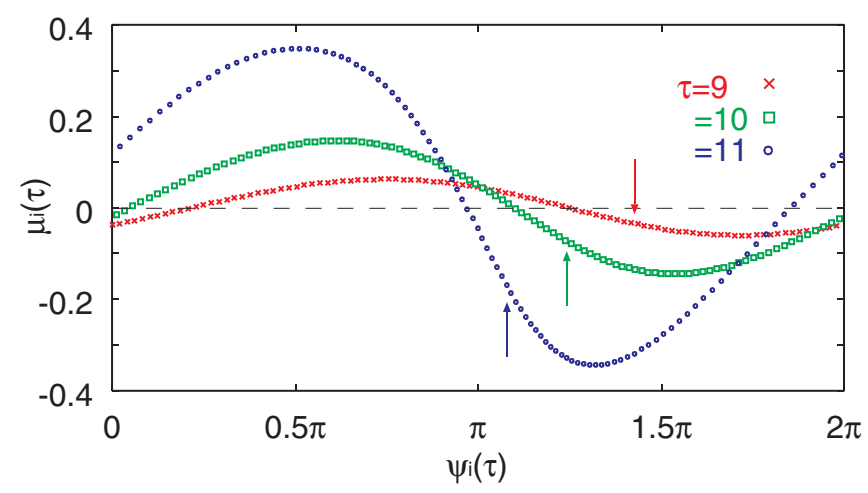

FIG. 2. (Color) Electron distributions in a phase plane of $\psi_{i}(\tau)=\psi_{i}(0)+\Delta \psi_{i}(\tau)$ and $\mu_{i}(\tau)$ derived from Eqs. (12) and (13) when $\tau=9$ (crosses), 10 (open squares), 11 (open circles). The arrow indicates the microbunch center which electrons within $\lambda_{r}$ are concentrated around. The initial amplitude of the radiation $|a(0)|=3.79 \times 10^{-5}$ is used.

the optical field is amplified. The position of the microbunch center is unclear only from Fig. 2 without Eqs. (12) and (13).

An advantage of the phase space used in Fig. 1 over the usual phase space is that the ellipse size clearly shows how much electrons shift from their initial phases. Another advantage is that one can see the distance between the microbunch center and the resonant electron. The longitudinal phase space normalized by FEL amplitude, which is composed of

$$
\Delta \Psi_{i}(\tau)=\Delta \psi_{i}(\tau) \frac{3}{|a(0)| e^{\sqrt{3} \tau / 2}}
$$

and

$$
M_{i}(\tau)=\mu_{i}(\tau) \frac{3}{|a(0)| e^{\sqrt{3} \tau / 2}},
$$

is useful for this purpose and is shown in Fig. 3. The shape of the distribution gradually changes when $\tau<4$ and remains almost constant in the high gain regime defined by $\tau \geq 4$. The microbunch center is close to the resonant electron when $\tau \ll 4$, but the space between them increases with $\tau$ and remains constant when $\tau \geq 4$.

In the high gain regime, the exponentially growing terms dominates in Eqs. (11)-(13), and the field is given by

$$
a(\tau) \sim \frac{|a(0)|}{3} e^{\sqrt{3} \tau / 2} e^{i \phi(\tau)},
$$

where $\phi(\tau)=\tau / 2+\phi(0)$. The energy and phase of the $i$ th electron are, respectively, given by

$$
\begin{gathered}
\mu_{i}(\tau) \sim \frac{2|a(0)| e^{\sqrt{3} \tau / 2}}{3} \cos \left[\psi_{i}(0)+\phi(\tau)-\pi / 6\right], \\
\Delta \psi_{i}(\tau) \sim \frac{2|a(0)| e^{\sqrt{3} \tau / 2}}{3} \cos \left[\psi_{i}(0)+\phi(\tau)-\pi / 3\right] .
\end{gathered}
$$

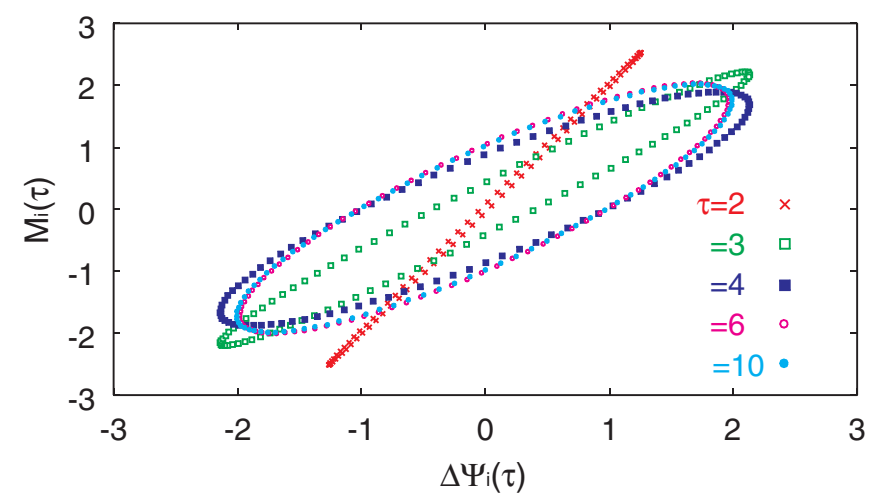

FIG. 3. (Color) Electron distributions in a normalized phase plane of $\Delta \Psi_{i}(\tau)$ given by Eq. (15) and $M_{i}(\tau)$ given by Eq. (16) for $\tau=2,3,4,6,10$. The center of the electron microbunch is located at $\left[\Delta \Psi_{i}(\tau), M_{i}(\tau)\right]=(0,-1)$ when $\tau \geq 4$.

The constant ellipse seen in Fig. 3 is $\Delta \Psi_{i}(\tau)^{2}+M_{i}(\tau)^{2}-$ $\sqrt{3} \Delta \Psi_{i}(\tau) M_{i}(\tau)=1$ when $\tau \geq 4$. The ellipse rotates clockwise as $\phi(\tau)$ increases linearly with $\tau$, while its size remains constant. This shows that the electron microbunching develops exponentially in size proportional to the FEL amplitude and the position of the bunch center shifts linearly proportional to the FEL phase. The microbunch center in the high gain regime is located at $\left[\Delta \Psi_{i}(\tau), M_{i}(\tau)\right]=(0,-1)$ in Fig. 3. The electrons inside the microbunch are thus concentrated around $\psi_{i}(0)+$ $\phi(\tau)-\pi / 3=\pi / 2$. The exponential decrease of the energy of the microbunch center corresponds to the exponential decrease of the energy of the microbunch as a whole. The energy radiated by the microbunch is used for the field amplification.

The field gain given by Eq. (14) can be used as long as $\left|\Delta \psi_{i}(\tau)\right| \ll 1$. However, the gain deviates from Eq. (14) near saturation, where the amplitude grows and $\left|\Delta \psi_{i}(\tau)\right| \ll 1$ does not hold any more for some electrons. The threshold amplitude above which Eq. (14) does not hold can be roughly estimated from calculation of Eq. (5). Substitution of Eqs. (17) and (19) into Eq. (5) gives

$$
\begin{aligned}
\frac{d|a(\tau)|}{d \tau}= & -\left\langle\operatorname { c o s } \left\{\psi_{i}(0)+\phi(\tau)+2|a(\tau)|\right.\right. \\
& \left.\left.\times \cos \left[\psi_{i}(0)+\phi(\tau)-\pi / 3\right]\right\}\right\rangle_{\zeta_{i}(\tau)=\zeta}, \\
\frac{d \phi(\tau)}{d \tau}= & \frac{1}{|a(\tau)|}\left\langle\operatorname { s i n } \left\{\psi_{i}(0)+\phi(\tau)+2|a(\tau)|\right.\right. \\
& \left.\left.\times \cos \left[\psi_{i}(0)+\phi(\tau)-\pi / 3\right]\right\}\right\rangle_{\zeta_{i}(\tau)=\zeta} .
\end{aligned}
$$

The values of the right hand sides of Eqs. (20) and (21) are calculated as a function of the value of $|a(\tau)|$ and shown in Figs. 4(a) and 4(b), respectively. In the calculation the value of $\psi_{i}(0)+\phi(\tau)$ is uniform over $2 \pi$. The solid line in (a) stands for Eq. (20) and the dash-dotted line in (b) for Eq. (21). The dotted lines in (a) and (b) are derived from 

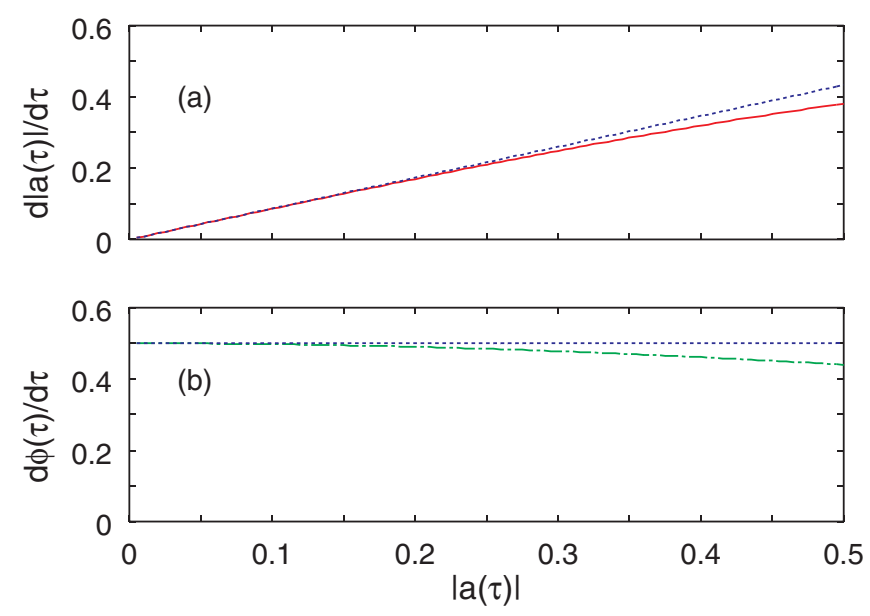

FIG. 4. (Color) The amplitude gain at time $\tau, d|a(\tau)| / d \tau$, calculated from Eq. (20) is given by the solid curve in (a) as a function of $|a(\tau)|$. The phase shift $d \phi(\tau) / d \tau$ calculated from Eq. (21) is represented by the dash-dotted line in (b). The dotted lines in (a) and (b) stand for Eq. (14).

Eq. (14). From the figure one can find that Eqs. (11)-(13) can be used when $|a(\tau)| \ll 1$.

\section{SATURATION}

One can calculate the efficiency and amplitude near saturation by starting with the initial values given by Eqs. (17)-(19) and numerically solving the timedependent 1D FEL equations. The initial field at an arbitrary time $\tau_{x}$ in the linear regime, where $\left|a\left(\tau_{x}\right)\right|=x \ll 1$, is given from Eq. (17) by

$$
a\left(\tau_{x}\right) \sim \frac{|a(0)|}{3} e^{\sqrt{3} \tau_{x} / 2} e^{i \phi\left(\tau_{x}\right)}=x e^{i \phi\left(\tau_{x}\right)} .
$$

The energy and phase of the $i$ th electron at $\tau_{x}$ are derived from substitution of Eq. (22) into Eqs. (18) and (19) and are, respectively, given by

$$
\begin{gathered}
\mu_{i}\left(\tau_{x}\right) \sim 2 x \cos \left[\psi_{i}(0)+\phi\left(\tau_{x}\right)-\pi / 6\right], \\
\psi_{i}\left(\tau_{x}\right) \sim \psi_{i}(0)+2 x \cos \left[\psi_{i}(0)+\phi\left(\tau_{x}\right)-\pi / 3\right] .
\end{gathered}
$$

The 1D FEL equations when $\tau \geq \tau_{x}$ are rewritten as follows:

$$
\begin{gathered}
\frac{d \mu_{i}(\tau)}{d \tau}=2|a(\tau)| \cos \left[\psi_{i}(\tau)+\phi(\tau)\right], \\
\frac{d \psi_{i}(\tau)}{d \tau}=\mu_{i}(\tau), \\
\frac{d|a(\tau)|}{d \tau}=-\left\langle\cos \left[\psi_{i}(\tau)+\phi(\tau)\right]\right\rangle_{\zeta_{i}=\zeta}, \\
\frac{d \phi(\tau)}{d \tau}=\frac{1}{|a(\tau)|}\left\langle\sin \left[\psi_{i}(\tau)+\phi(\tau)\right]\right\rangle_{\zeta_{i}=\zeta}
\end{gathered}
$$

Using Eqs. (22)-(24) as the initial values and solving Eqs. (25)-(28) numerically, $d|a(\tau)| / d \tau$ and $d \phi(\tau) / d \tau$ can be obtained as a function of $\tau-\tau_{x}$ in Fig. 5. In the calculation, $x=0.1$ is used as an example. It is found that $d|a(\tau)| / d \tau$ decreases down to 0 when $\tau_{p}-\tau_{0.1}=3.7$. Here $\tau_{p}$ is the time when the efficiency and amplitude reach their peaks and the amplitude gain turns to negative. Integration of $d|a(\tau)| / d \tau$ from $\tau_{0.1}$ to $\tau_{p}$ yields

$$
\int_{\tau_{0.1}}^{\tau_{p}} \frac{|a(\tau)|}{d \tau} d \tau=1.08
$$

The peak amplitude is thus given by

$$
\left|a\left(\tau_{p}\right)\right|=1.18,
$$

which agrees well with the peak amplitude of the SASE in the steady-state regime obtained in a numerical calculation [6]. The value in the right hand side of Eq. (29) does not depend on the value of $x$ as long as $x \ll 1$.

The efficiency is obtained from Eq. (29). The energy carried by the intracavity radiation over unit length of $\lambda_{r}$ along the propagation direction is given from Eq. (2) by

$$
\epsilon_{0} \lambda_{r} \Sigma E(\zeta, \tau)^{2}=\frac{\epsilon_{0} \lambda_{r} \Sigma \gamma_{0}^{4} m^{2} c^{4}|a(\zeta, \tau)|^{2}(4 \pi \rho)^{4}}{4 \pi^{2} e^{2} a_{w}^{2} \lambda_{w}^{2} F^{2}}
$$

where $\Sigma$ is the effective radiation area averaged over the length of the undulator. The time derivative of the intracavity energy, $\partial\left[\epsilon_{0} \lambda_{r} \Sigma E(\zeta, \tau)^{2}\right] / \partial \tau$ is equal to the amount of the instantaneous energy loss of the microbunch given by $-N_{e} m c^{2} d\left\langle\gamma_{i}(\tau)-\gamma_{0}\right\rangle_{\zeta_{i}(\tau)=\zeta} / d \tau$. Here $N_{e}=n_{e} \lambda_{r} \Sigma$ is the number of electrons contained in a unit volume of $\lambda_{r} \Sigma$. The electron beam density is given by $n_{e}=$ $\left(16 \rho^{3} \gamma_{0}^{3} \epsilon_{0} m c^{2} k_{w}^{2}\right) /\left(e^{2} a_{w}^{2} F^{2}\right)$ from Eq. (1), and thus

$$
N_{e}=(4 \pi \rho)^{3} \frac{\lambda_{r} \epsilon_{0} \Sigma \gamma_{0}^{3} m c^{2}}{\pi e^{2} a_{w}^{2} \lambda_{w}^{2} F^{2}}
$$

The average of the instantaneous energy loss of the microbunch at time $\tau$ is thus given by

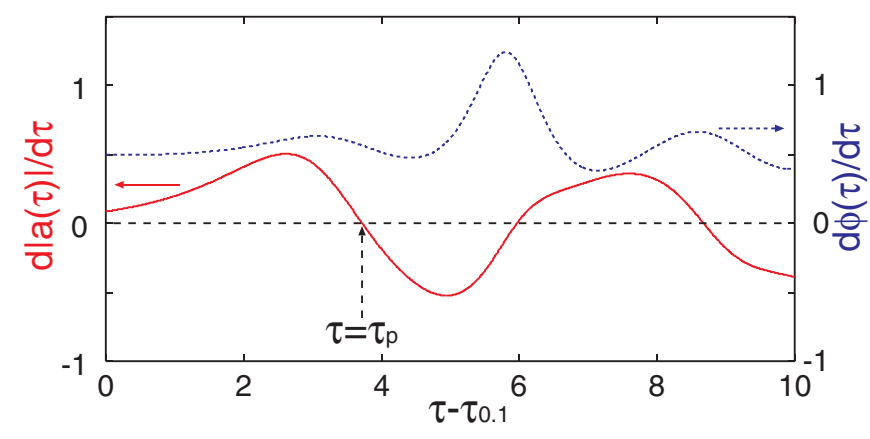

FIG. 5. (Color) The amplitude gain $d|a(\tau)| / d \tau$ (solid line) and phase shift $d \phi(\tau) / d \tau$ (dotted line) calculated numerically from the 1D FEL equations given by Eqs. (25)-(28) as a function of $\tau-\tau_{0.1}$. The gain is zero at $\tau_{p}$. The initial values for the calculation are given by Eqs. (22)-(24). 


$$
-\frac{d\left\langle\mu_{i}(\tau)\right\rangle_{\zeta_{i}(\tau)=\zeta}}{d \tau}=\frac{d|a(\tau)|^{2}}{d \tau} .
$$

Integration of Eq. (31) in $\tau$ yields

$$
-\left\langle\mu_{i}(\tau)\right\rangle_{\zeta_{i}(\tau)=\zeta}=|a(\tau)|^{2}
$$

The extraction efficiency for the microbunch at time $\tau$ is given by $-\rho\left\langle\mu_{i}(\tau)\right\rangle_{\zeta_{i}(\tau)=\zeta}$ and is almost equivalent to the efficiency $\eta(\tau)$ for the electron beam composed of many microbunches. This is because all the microbunches interact with almost the same steady-state field in a SASE FEL. Substitution of Eq. (29) into Eq. (32) leads to the efficiency at $\tau_{p}$

$$
\eta\left(\tau_{p}\right)=1.39 \rho,
$$

which agrees well with $\eta=1.37 \rho$ obtained in a numerical simulation [7] and with $\eta \approx \rho$ obtained in the theoretical work based on the coupled Klimontovich-Maxwell equations [5].

The number of undulator periods $N_{w}$ required for the saturation to occur depends on the value of $\tau_{x=0.1}$, while $\tau_{p}-\tau_{x=0.1}$ is derived to be 3.7 from Fig. 5. The time $\tau_{x=0.1}$ will be derived from Eq. (22) when the initial amplitude $|a(0)|$ is obtained. The amplitude $|a(0)|$ is equivalent to the amplitude of spontaneous radiation emitted in about one gain length [5] and is estimated to be $\sqrt{2 \pi \rho / N_{e}}$ from Eq. (5), since the variance of $\left\langle\cos \left[\psi_{i}(\tau)\right]\right\rangle_{\zeta_{i}(\tau)=\zeta}$ per the cooperation length is given by $(4 \pi \rho) /\left(2 N_{e}\right)$ from a statistical consideration [14]. Substitution of Eq. (30) into $|a(0)|=\sqrt{2 \pi \rho / N_{e}}$ gives

$$
|a(0)|=\frac{1}{\rho} \frac{e}{\sqrt{32 \pi m c^{2} \varepsilon_{0}}} \frac{F a_{w} \lambda_{w}}{\sqrt{\gamma_{0}^{3} \Sigma \lambda_{r}}} .
$$

The effective radiation area is $\Sigma=\lambda_{r} Z_{R} / 2$ where $Z_{R}=$ $R \lambda_{w} /(4 \pi \rho)$ is the Rayleigh range and $R \geq 1$ for 1D SASE FEL theory [5]. Substitution of $\Sigma=R \lambda_{w} \lambda_{r} /(8 \pi \rho)$ into Eq. (34) yields $|a(0)|=P / \sqrt{\rho R}$, where

$$
P=\frac{e}{\sqrt{m c^{2} \varepsilon_{0}}} \sqrt{\frac{F^{2} a_{w}^{2} \gamma_{0}}{\lambda_{w}\left(1+a_{w}^{2}\right)^{2}}} .
$$

Substitution of $|a(0)|=P / \sqrt{\rho R}$ into Eq. (22) when $x=$ 0.1 gives

$$
\frac{\sqrt{3}}{2} \tau_{0.1}=-1.2-\ln (P)+\frac{1}{2} \ln (\rho R) .
$$

This equation yields

$$
\tau_{p}=\frac{2}{\sqrt{3}}\left[-1.2-\ln (P)+\frac{1}{2} \ln (\rho R)\right]+3.7 .
$$

The parameter $P$ ranges from $1 \times 10^{-6}$ to $2 \times 10^{-5}$ for almost all the Compton FELs [15], $\rho$ ranges from 0.001 to 0.01 , and $R$ is order of unity. The time $\tau_{p}$ is estimated to range from 11 to 16 . This is consistent with the well known saturation condition of a SASE FEL: $\rho N_{w} \approx 1$ [5].

\section{NUMERICAL SIMULATION}

In order to confirm the present analytical solutions for the electron phase space evolution, a numerical simulation is performed which solves the 1D FEL equations in a timedependent manner [16] and takes into account a shot-noise effect by employing a method by Penman and McNeil [14]. The electron bunch length $L_{b}$ and slippage length $N_{w} \lambda_{r}$ used in the simulation are both $20 \lambda_{r} /(4 \pi \rho)$, and the FEL parameter is $\rho=0.0045$. The parameters used in the simulation such as $a_{w}, \lambda_{w}$, and $\gamma_{0}$ yield $P=2.54 \times$ $10^{-6}$ and $P / \sqrt{\rho}=3.79 \times 10^{-5}$, which is the same as the initial amplitude $|a(0)|$ used in Figs. 1 and 2. These parameters ensure saturation within a single pass.

Figure 6 shows the calculated electron distributions of an electron microbunch in a longitudinal phase space of $\Delta \Psi_{i}(\tau)$ and $M_{i}(\tau)$, which is initially located at $\zeta_{i}(0)=$ -1.7 . The distribution is similar to that shown in Fig. 3. The time evolution in a phase space of $\Delta \psi_{i}(\tau)$ and $\mu_{i}(\tau)$ shown in Fig. 7 is also similar to that shown in Fig. 1, although the size of the ellipse in Fig. 7 is roughly twice smaller than that in Fig. 1 despite the same $\tau$. The size difference can be attributed to the time required for the spectrum narrowing. This effect is not included in the present analytical study, but in the simulation. The initial field at $\tau=0$ is approximated by $a(0)=|a(0)| e^{i \phi(0)}$ in the present analysis, though the spectrum narrowing is established after some undulator periods $[8,12]$.

Electron microbunches with initial positions different from $\zeta_{i}(0)=-1.7$ also show similar evolution to that with $\zeta_{i}(0)=-1.7$. This is because each microbunch is modulated by the steady-state field, which has almost the same field envelope independent of $\zeta$. The energy and

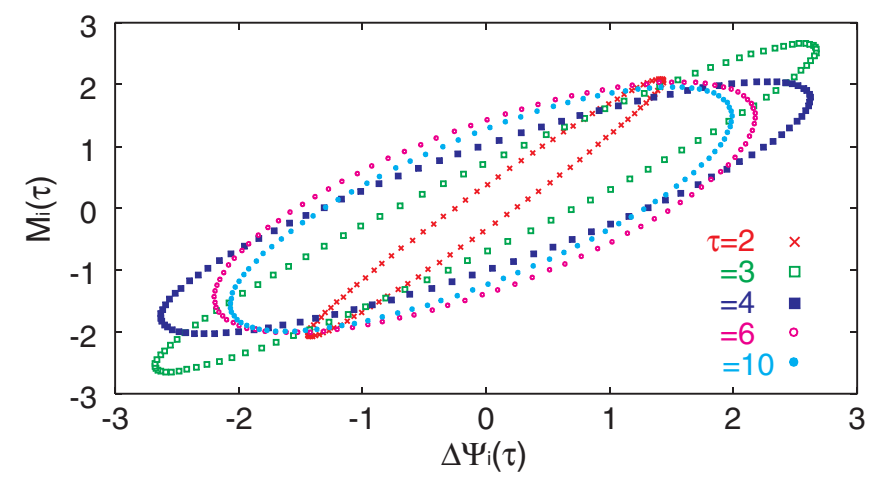

FIG. 6. (Color) Electron distributions in a phase plane of $\Delta \psi_{i}(\tau)$ and $M_{i}(\tau)$ for an electron microbunch whose initial position is $\zeta_{i}(0)=-1.7$. The distributions are obtained in a time-dependent numerical simulation. The electron bunch and slippage lengths used in the simulation are both $20 \lambda_{r} /(4 \pi \rho)$, the FEL parameter $\rho=0.0045$. The parameters used in the simulation give $P=$ $2.54 \times 10^{-6}$. 


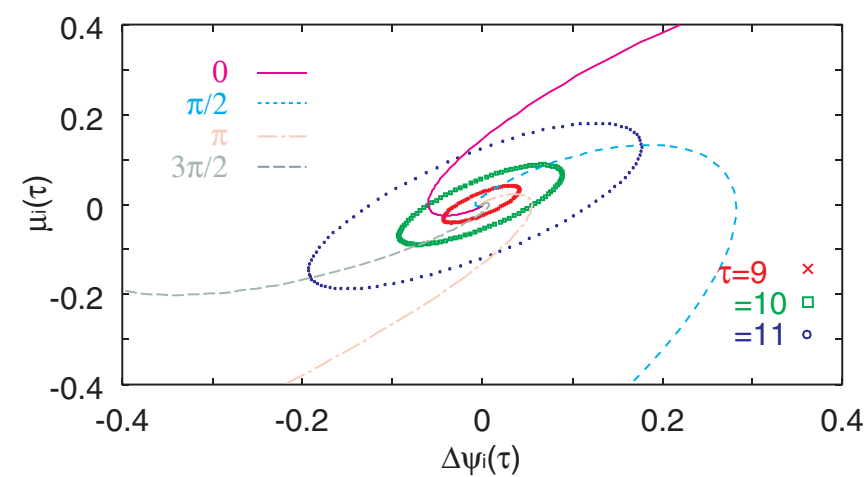

FIG. 7. (Color) Electron distributions of an electron microbunch with $\zeta_{i}(0)=-1.7$ in a phase plane of $\Delta \psi_{i}(\tau)$ and $\mu_{i}(\tau)$ obtained from a time-dependent simulation when $\tau=9$ (solid line), 10 (dotted line), 11 (dash-dotted line). The simulation parameters are the same as in Fig. 6.

phase modulation of each microbunch are similar to each other and given by Eqs. (12) and (13). The maximum efficiency obtained in the simulation is $\eta_{\max }=0.0053$ when $\tau \approx 15.0$, as shown in Fig. 8, while Eqs. (33) and (35) give the peak efficiency of $\eta\left(\tau_{p}\right)=0.0063$ at $\tau_{p}=$ 14.1 when $\rho=0.0045$ and $P=2.54 \times 10^{-6}$. The present study is almost consistent with the result of the numerical simulation.

The electron phase space evolution before saturation studied in the present numerical simulation does not depend on the electron pulse length or the slippage length as far as both of them are longer than $\lambda_{r} \tau_{p} /(4 \pi \rho)$. This corresponds to the shortest slippage length necessary for the steady-state field to reach saturation. The shortest slippage length is about $15 \lambda_{r} /(4 \pi \rho)$ in the simulation shown in Fig. 8.

\section{COHERENT OPTICAL TRANSITION RADIATION FROM ELECTRON MICROBUNCHING}

The evolution of electron beam microbunching in a SASE FEL has been experimentally studied by means of coherent OTR at APS [9]. They have found that the coherent OTR intensity at FEL wavelength increases as a function of the undulator length proportionally to the FEL

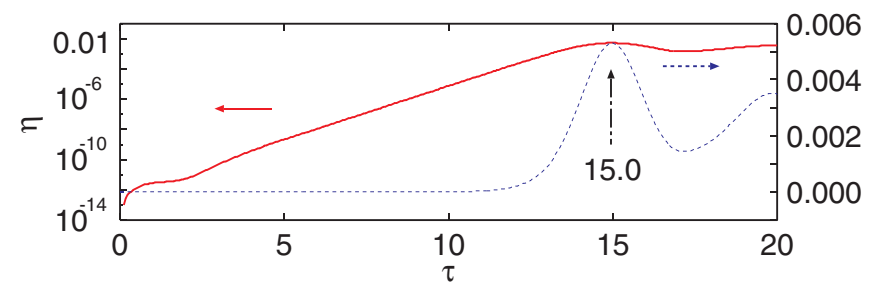

FIG. 8. (Color) Efficiency as a function of $\tau$ obtained in a simulation. The solid line is semilog plot, and the dotted line is linear plot. The simulation parameters are the same as in Fig. 6. power. This experimental study has been supported by theoretical studies where the electron beam pulse after FEL interaction is assumed to have a Gaussian distribution modulated sinusoidally at FEL wavelength and its harmonics as follows $[17,18]$ :

$$
h(z)=\frac{1}{\sqrt{2 \pi} \sigma} \exp \left(-\frac{z^{2}}{2 \sigma^{2}}\right)\left[1+\sum_{n=1}^{\infty} b_{n} \cos \left(n k_{r} z\right)\right]
$$

where $\sigma$ is the rms longitudinal electron pulse length and $b_{n}$ is bunching parameter of the $n$th harmonic of FEL. This equation yields narrow spikes at the fundamental FEL wavelength and its harmonics and explains the coherent OTR experiments performed at APS [9]. However, it is not straightforward to connect the bunching parameter $b_{n}$ with such parameters as the FEL amplitude. In this section, the coherent OTR intensity is shown to be proportional to the FEL intensity without an assumption used in Eq. (37), but with the present analytical solutions of electron phase space evolution.

The radiation field at a frequency $\omega=2 \pi c / \lambda$ produced from a single electron is proportional to $\mathcal{E}_{k} \propto e^{i\left[\omega t+\varphi_{k}\right]}$ $[19,20]$, where

$$
\varphi_{k}=\frac{2 \pi z_{k}}{\lambda}
$$

describes the position of the $k$ th electron with respect to the center of the electron beam pulse, and $z_{k}$ is the distance of the $k$ th electron from the center of the beam pulse along the propagation direction. When the particle number of the electron beam is $\tilde{N}_{e}$, the radiation power $P(\omega)$ at the frequency $\omega$ is proportional to [19]

$$
P(\omega)=\sum_{k, j}^{\tilde{N}_{e}} \mathcal{E}_{k} \mathcal{E}_{j}^{*} \propto \tilde{N}_{e}+\sum_{k \neq j}^{\tilde{N}_{e}} e^{i\left(\varphi_{k}-\varphi_{j}\right)} .
$$

The first term in the right-hand side of Eq. (39) represents incoherent emission and the second term coherent emission.

The analytical form of coherent OTR spectrum can be obtained from Eq. (39), when the analytical form of the $k$ th electron position $z_{k}$ is available. This electron position during SASE evolution has been obtained in Sec. III when the incident electron pulse has a rectangular shape and is given from Eq. (19) by

$$
\begin{aligned}
z_{k}(\tau) & =\frac{\lambda_{r}}{2 \pi}\left[\psi_{k}(0)+\Delta \psi_{k}(\tau)\right] \\
& =\frac{\lambda_{r}}{2 \pi} \psi_{k}(0)+\frac{\lambda_{r}}{2 \pi} 2|a(\tau)| \cos \left[\psi_{k}(0)+\phi(\tau)-\pi / 3\right],
\end{aligned}
$$

in the high gain regime $\tau \geq 4$ before saturation $|a(\tau)| \ll$ 1. When the rectangular electron pulse length is assumed to be $l \lambda_{r}$, the total electric field is proportional to 


$$
\begin{aligned}
\frac{1}{\tilde{N}_{e}} \sum_{k}^{\tilde{N}_{e}} \mathcal{E}_{k} & \propto \frac{1}{l \lambda_{r}} \int_{-l \lambda_{r} / 2}^{l \lambda_{r} / 2} e^{i(\omega t+2 \pi z / \lambda)} d z \approx \frac{e^{i \omega t}}{2 \pi l} \int_{-\pi l}^{\pi l} e^{i\left(\lambda_{r} / \lambda\right) \psi}\left\{1+i \frac{\lambda_{r}}{\lambda} 2|a(\tau)| \cos [\psi+\phi(\tau)-\pi / 3]\right\} d \psi \\
& =e^{i \omega t}\left(\frac{\sin \left(\pi l \lambda_{r} / \lambda\right)}{\pi l \lambda_{r} / \lambda}+i \frac{\lambda_{r}}{\lambda}|a(\tau)|\left\{e^{-i[\phi(\tau)-\pi / 3]} \frac{\sin \left[\pi l\left(1-\lambda_{r} / \lambda\right)\right]}{\pi l\left(1-\lambda_{r} / \lambda\right)}+e^{i[\phi(\tau)-\pi / 3]} \frac{\sin \left[\pi l\left(1+\lambda_{r} / \lambda\right)\right]}{\pi l\left(1+\lambda_{r} / \lambda\right)}\right\}\right) .
\end{aligned}
$$

From Eq. (41), the coherent OTR power spectrum $P_{c}(\omega)$ is proportional to

$$
\begin{aligned}
P_{c}(\omega) \propto & \frac{1}{\tilde{N}_{e}\left(\tilde{N}_{e}-1\right)} \sum_{k \neq j}^{\tilde{N}_{e}} \mathcal{E}_{k} \mathcal{E}_{j}^{*} \\
= & \frac{\sin ^{2}\left(\pi l \lambda_{r} / \lambda\right)}{\left(\pi l \lambda_{r} / \lambda\right)^{2}}+2|a(\tau)| \frac{\sin \left(\pi l \lambda_{r} / \lambda\right)}{\pi l} \sin [\phi(\tau)-\pi / 3]\left\{-\frac{\sin \left[\pi l\left(1+\lambda_{r} / \lambda\right)\right]}{\pi l\left(1+\lambda_{r} / \lambda\right)}+\frac{\sin \left[\pi l\left(1-\lambda_{r} / \lambda\right)\right]}{\pi l\left(1-\lambda_{r} / \lambda\right)}\right\} \\
& +\left(\frac{\lambda_{r}}{\lambda}\right)^{2}|a(\tau)|^{2}\left\{\frac{\sin ^{2}\left[\pi l\left(1-\lambda_{r} / \lambda\right)\right]}{\left[\pi l\left(1-\lambda_{r} / \lambda\right)\right]^{2}}+\frac{\sin ^{2}\left[\pi l\left(1+\lambda_{r} / \lambda\right)\right]}{\left[\pi l\left(1+\lambda_{r} / \lambda\right)\right]^{2}}+2 \cos [2 \phi(\tau)-2 \pi / 3] \frac{\sin \left[\pi l\left(1-\lambda_{r} / \lambda\right)\right]}{\pi l\left(1-\lambda_{r} / \lambda\right)}\right. \\
& \left.\times \frac{\sin \left[\pi l\left(1+\lambda_{r} / \lambda\right)\right]}{\pi l\left(1+\lambda_{r} / \lambda\right)}\right\} .
\end{aligned}
$$

The first term in the right hand side of Eq. (42) shows well known coherent OTR produced from the electron beam pulse length $[19,21]$. The second term is negligible, since it is much smaller than unity due to $\left[\sin \left(\pi l \lambda_{r} / \lambda\right) / \pi l\right] \ll 1$. The brace in the third term has three terms. Among them, only the first term can approach unity when $\lambda \approx \lambda_{r}$. Finally, Eq. (42) can be approximated by

$$
\begin{aligned}
P_{c}(\omega) \propto & \frac{\sin ^{2}\left(\pi l \lambda_{r} / \lambda\right)}{\left(\pi l \lambda_{r} / \lambda\right)^{2}} \\
& +\left(\frac{\lambda_{r}}{\lambda}\right)^{2}|a(\tau)|^{2} \frac{\sin ^{2}\left[\pi l\left(1-\lambda_{r} / \lambda\right)\right]}{\left[\pi l\left(1-\lambda_{r} / \lambda\right)\right]^{2}} .
\end{aligned}
$$

The second term shows the coherent OTR from SASE induced electron microbunching and tells us not only that this radiation appears at FEL wavelength but also that its power is proportional to the square of the normalized FEL amplitude, that is the FEL optical amplitude [see Eq. (2)]. The APS experiment, where dependence of coherent OTR on the undulator length is found to be similar to that of SASE power [9], can be explained directly from Eq. (43) without assuming such electron distribution as given by Eq. (37).

\section{CONCLUSION}

The phase space evolution of electrons in a SASE FEL operating in the linear regime before saturation in the resonant case has been solved analytically from the 1D FEL equation. The evolutions of the phase change $\Delta \psi_{i}(\tau)$ and energy change $\mu_{i}(\tau)$ of the $i$ th electron are, respectively, represented by the sum of three independent analytical solutions similarly to the evolution of the SASE field; an exponentially growing term, an exponentially decaying term, and an oscillating term. The electrons are shown to be bunched around $\pi / 6$ ahead of a resonant electron every resonant FEL wavelength in the high gain regime, which explains the positive FEL gain. A newly introduced longitudinal phase space composed of $\Delta \psi_{i}(\tau)$ and $\mu_{i}(\tau)$ allows one to see how much electron microbunching develops and where the microbunch center is. The electron distribution in the phase space expands exponentially with time in size, rotating clockwise linearly in the high gain regime defined by $\tau \geq 4$. These expansion in size and clockwise rotation correspond to the exponential increase of the amplitude and linear increase of the phase of the SASE field, respectively, and represent the time evolution of the electron microbunching. The microbunch center is located where $\Delta \psi_{i}(\tau)=0$ and $\mu_{i}(\tau)<0$, and the energy of the microbunch center decreases exponentially. The present analytical solutions will be applied to a study of the electron microbunching induced by FEL interaction. The coherent OTR intensity at FEL wavelength produced from electron microbunching has been shown to be proportional to FEL intensity.

\section{ACKNOWLEDGMENTS}

The author would like to acknowledge valuable discussions with S. Hiramatsu at KEK, H. Hama at Tohoku University, E. Minehara at JAERI, and S. Benson at Jefferson Lab.

[1] V. Ayvazyan, N. Baboi, I. Bohnet, R. Brinkmann, M. Castellano, P. Castro, L. Catani, S. Choroba, A. Cianchi, M. Dohlus, H. T. Edwards, B. Faatz, A. A. Fateev, J. Feldhaus, K. Flöttmann, A. Gamp, T. Garvey, H. Genz, Ch. Gerth, V. Gretchko, B. Grigoryan, U. Hahn, C. Hessler, K. Honkavaara, M. Hüning, R. Ischebeck, M. Jablonka, T. Kamps, M. Körfer, M. Krassilnikov, J. Krzywinski, M. Liepe, A. Liero, T. Limberg, H. Loos, M. Luong, C. Magne, J. Menzel, P. Michelato, M. Minty, U.-C. Müller, D. Nölle, A. Novokhatski, C. Pagani, F. 
Peters, J. Pflüger, P. Piot, L. Plucinski, K. Rehlich, I. Reyzl, A. Richter, J. Rossbach, E. L. Saldin, W. Sandner, H. Schlarb, G. Schmidt, P. Schmüser, J. R. Schneider, E. A. Schneidmiller, H.-J. Schreiber, S. Schreiber, D. Sertore, S. Setzer, S. Simrock, R. Sobierajski, B. Sonntag, B. Steeg, F. Stephan, K. P. Sytchev, K. Tiedtke, M. Tonutti, R. Treusch, D. Trines, D. Türke, V. Verzilov, R. Wanzenberg, T. Weiland, H. Weise, M. Wendt, I. Will, S. Wolff, K. Wittenburg, M. V. Yurkov, and K. Zapfe, Phys. Rev. Lett. 88, 104802 (2002).

[2] C. Limborg, Nucl. Instrum. Methods Phys. Res., Sect. A 507, 378 (2003).

[3] T. Shintake, T. Tanaka, T. Hara, K. Togawa, T. Inagaki, Y. J. Kim, T. Ishikawa, H. Kitamura, H. Baba, H. Matsumoto, S. Takeda, M. Yoshida, and Y. Takasu, Nucl. Instrum. Methods Phys. Res., Sect. A 507, 382 (2003).

[4] Norman M. Kroll and Wayne A. McMullin, Phys. Rev. A 17, 300 (1978).

[5] Kwang-Je Kim, Phys. Rev. Lett. 57, 1871 (1986);Nucl. Instrum. Methods Phys. Res., Sect. A 250, 396 (1986); Kwang-Je Kim and Ming Xie, ibid. 331, 359 (1993).

[6] R. Bonifacio, F. Casagrande, G. Oerchioni, L. De Salvo Souza, P. Pierini, and N. Piovella, Riv. Nuovo Cimento 13, 1 (1990); R. Bonifacio, L. De Salvo Souza, P. Pierini, and N. Piovella, Nucl. Instrum. Methods Phys. Res., Sect. A 296, 358 (1990).

[7] E. L. Saldin, E. A. Schneidmiller, and M. V. Yurkov, in The Physics of Free Electron Lasers (Springer, Berlin, 2000), pp. $48-54$.

[8] W. B. Colson, in Laser Handbook, edited by W. B. Colson, C. Pellegrini, and A. Renieri (North Holland, Amsterdam, 1990), Vol. 6, pp. 115-193.

[9] A. H. Lumpkin, R. Dejus, W. J. Berg, M. Borland, Y.C. Chae, E. Moog, N. S. Sereno, and B. X. Yang, Phys. Rev.
Lett. 86, 79 (2001); A. H. Lumpkin, R. Dejus, J. W. Lewellen, W. Berg, S. Biedron, M. Borland, Y. C. Chae, M. Erdmann, Z. Huang, K.-J. Kim, Y. Li, S. V. Milton, E. Moog, D. W. Rule, V. Sajaev, and B.X. Yang, ibid. 88, 234801 (2002).

[10] C. A. Brau, Free-Electron Lasers (Academic, San Diego, 1990).

[11] W. B. Colson and S. K. Ride, Phys. Lett. 76A, 379 (1980).

[12] N. Nishimori, R. Hajima, R. Nagai, and E. J. Minehara, Nucl. Instrum. Methods Phys. Res., Sect. A 507, 79 (2003).

[13] W. B. Colson, J. C. Gallardo, and P. M. Bosco, Phys. Rev. A 34, 4875 (1986).

[14] C. Penman and B.W. J. McNeil, Opt. Commun. 90, 82 (1992).

[15] W. B. Colson, in Proceedings of the 23rd International Free Electron Laser Conference (FEL2001), Darmstadt, Germany, 2001 (Elsevier, Amsterdam, 2002), p. II-67.

[16] R. Hajima, N. Nishimori, R. Nagai, and E. J. Minehara, Nucl. Instrum. Methods Phys. Res., Sect. A 475, 270 (2001).

[17] J. Rosenzweig, G. Travish, and A. Tremaine, Nucl. Instrum. Methods Phys. Res., Sect. A 365, 255 (1995); A. Tremaine, J. B. Rosenzweig, S. Anderson, P. Frigola, M. Hogan, A. Murokh, C. Pellegrini, D. C. Nguyen, and R. L. Sheffield, Phys. Rev. Lett. 81, 5816 (1998).

[18] C. J. Hirschmugl, M. Sagurton, and G. P. Williams, Phys. Rev. A 44, 1316 (1991).

[19] H. Wiedemann, Particle Accelerator Physics I (Springer, Berlin, 1993), pp. 300-336.

[20] Y. Shibata, T. Takahashi, T. Kanai, K. Ishi, M. Ikezawa, J. Ohkuma, S. Okuda, and T. Okada, Phys. Rev. E 50, 1479 (1994).

[21] John S. Nodvick and David S. Saxon, Phys. Rev. 96, 180 (1954). 